\title{
Large plexiform neurofibroma on right thigh of a farmer in the course of Recklinghausen disease - Case report
}

\author{
Jacek Sompor ${ }^{1}$, Andrzej Prystupa ${ }^{2}$, Bartłomiej Wawrzycki ${ }^{3}$, Katarzyna Wojciechowska ${ }^{4}$, \\ Emila Kancik², Ewa Kurys-Denis ${ }^{5}$, Andrzej Ochal', Tomasz Prystupa ${ }^{6}$, Mariusz Jojczuk', \\ Tomasz Kulesza', Halina Antosz ${ }^{4}$ \\ ${ }^{1}$ Department of Trauma Surgery and Emergency Medicine, Medical University of Lublin, Poland \\ ${ }^{2}$ Department of Internal Medicine, Medical University of Lublin, Poland \\ ${ }^{3}$ Department of Dermatology, Venerology and Paediatric Dermatology, Medical University of Lublin, Poland \\ ${ }^{4}$ Department of Clinical Genetics, Medical University of Lublin, Poland \\ ${ }^{5}$ II Department of Medical Radiology, Medical University of Lublin, Poland \\ ${ }^{6}$ Students Research Association, Medical University of Lublin, Poland
}

Sompor J, Prystupa A, Wawrzycki B, Wojciechowska K, Kancik E, Kurys-Denis E, Ochal A, Prystupa T, Jojczuk M, Kulesza T, Antosz H. Large plexiform neurofibroma on right thigh of a farmer in the course of Recklinghausen disease - Case report. J Pre-Clin Clin Res. 2014; 8(2): 109-112. doi: 10.5604/18982395.1135661

\section{Abstract}

A 59-year-old farmer was admitted to the Department of Trauma Surgery because of pain associated with the presence of a tumour on the side of the right thigh. The patient stated that his biggest problem was great difficulty in moving and handling agricultural machinery due to the aforementioned lesion. On physical examination, the patient presented with numerous cutaneous and subcutaneous neurofibromas, as well as one large plexiform neurofibroma. Diagnosis of Neurofibromatosis type 1 was established, based on National Institutes of Health diagnostic criteria. Moreover, molecular genetic testing found known pathogenic mutation p.Arg1769* in one of the alleles of NF1 gene (heterozygote), typical for Recklinghausen disease. The big plexiform neurofibroma in the patient was not operated on because of lung cancer.

\section{Key words}

Recklinghausen disease, Neurofibromatosis type 1, tumour of the right thigh

\section{CASE REPORT}

A 59-year-old farmer was admitted to the Department of Trauma Surgery because of pain associated with the presence of a tumour on the side of the right thigh. He also reported decreased exercise tolerance, dyspnoea at rest and progressive weakness. The patient stated that his biggest problem was the great difficulty in moving and handling of agricultural machinery due to the aforementioned lesion located on the right thigh.

According to the patient's medical records he has also been diagnosed with epilepsy and prostatic hyperplasia. The patient was not previously diagnosed because of these symptoms.

On physical examination, patient presented with numerous cutaneous and subcutaneous neurofibromas, as well as one large plexiform neurofibroma. Hundreds of dome-shaped, soft, skin-coloured to pinkish nodules of different sizes, varying between a few millimeters to 2.5-3 centimeters covered the patient's head, trunk and proximal extremities. Within the side surface of the right thigh there was also an extensive plexiform neurofibroma with overlying hyperpigmented skin, and 'bag of worms' feeling on palpation. Careful clinical investigation revealed café-aulait macules on the trunk and extremities with the largest

Address for correspondence: Andrzej Prystupa, Department of Trauma Surgery and Emergency Medicine, Medical University of Lublin, Staszica 16, 20-081 Lublin, Poland

E-mail: aprystup@mp.pl

Received: 22 March 2014; accepted: 20 November 2014 one reaching $15 \mathrm{~cm}$ of the longest diameter. The additionally presented with discrete freckling in the axillary folds which were further identified as a so-called Crowe sign.

On auscultation of the lung fields vesicular murmur was heard. The heart rate was regular at 100 beats per minute rate. Systolic murmur was audible over the mitral valve. Abdomen was soft, painless, with no peritoneal signs and no pathological masses sensed on deep palpation. The liver and spleen were not enlarged. Basic laboratory tests showed no abnormalities.

Ophthalmologic split-lamp examination revealed the presence of iris Lisch nodules - hamartomas, highly specific for von Recklinghausen disease. The consulting dermatologist confirmed the nature of the cutaneous lesions as neutrofibromas.

Diagnosis of Neurofibromatosis type 1 (NF-1) was established based on National Institutes of Health diagnostic criteria.

Moreover, molecular genetic testing found known pathogenic mutation p.Arg1769* in one of the alleles of NF-1 gene (heterozygote) typical for Recklinghausen disease. The sequence analysis of NF-1 gene (exons 1-58) with surrounding intronic sequences was performed. Nextgeneration sequencing (NGS) technology and the nextgeneration sequencing diagnostic panel were used for exonlevel analyses. Additionally, exons 7, 11, 38, 41 and 43 were analyzed by direct sequencing.

The patient had four brothers and one sister, but there was no knowledge of any close relative presenting the symptoms of Neurofibromatosis type I in his family. The mutation seemed to have arisen de novo in the patient's genome. 
Because of increased malignancy ratio in NF-1, an additional set of radiological examinations were performed. Abdominal ultrasound showed no abnormalities. Chest X-ray confirmed clinically suspected generalized, pulmonary emphysema and right-sided scoliosis, however with no other significant pathologies. Pelvis X-ray showed a big left-sided vertebral column rotoscoliosis involving a pathological rotation of the pelvis and both femur bones. Developed signs of degenerative bone and sacro-iliac joints disease were also observed. Histopathology of biopsied nodules presented the typical picture of neurofibromas.

The patient also underwent $\mathrm{CT}$ of the chest with intravenous contrast administration, which revealed a singular, irregularly outlined tissue area of $15 \times 17 \mathrm{~mm}$ localized in the second segment of the right lung, with radially oriented outgrowths extending in the direction of pulmonary parenchyma. The solitary tumour enhanced pathologically after intravenous contrast administration proved to belung cancer. Additionally, enlarged mediastinal lymph nodes were noted, being in favour of tumour lymph nodes metastasis. Other pathologies included multiple generalized lesions of emphysema and fibrotic changes in the pulmonary parenchyma. The abdominal CT with contrast showed, furthermore, the presence of an unclear, hypodense change in segment IVB of the liver, close to the hepatoumbilical ligament, which was completely invisible to the ultrasound examination. Head MRI did not show any pathological changes.

After pulmonary consultation, the patient was qualified for palliative chemotherapy because of the lung cancer.

\section{DISCUSSION}

Neurofibromatosis type I (NF-1) is inherited as an autosomal dominant disorder with complete penetrance and variable expression [1]. Birth incidence is $1 / 500$ with a prevalence of $1 / 4,000$ [2]. The NF-1 gene is located at chromosome 17q11.2 and contains 350 kilobases of DNA [3]. It has 60 exons and codes for an 11- to $13-\mathrm{kb}$ transcript with an open reading frame coding for 2,818 amino acids [4].

NF-1 gene encodes neurofibromin, which is a guanosine triphosphatase (GTPase)-activating protein (GAP) that helps maintain the proto-oncogene RAS in an inactive form. Loss of neurofibromin results in increased RAS activity, particularly in neurocutaneous tissues, leading to constitutive downstream signaling increased uncontrolled proliferation and tumourigenesis [6]. Dasgupta et al. [7] suggest that neurofibromin also can positively regulate cyclic adenosine monophosphate (AMP) levels. Increased cyclic AMP levels are associated with reduced cell growth.

Many different mutations within the NF-1 gene can cause Neurofibromatosis type 1 . The mutational spectrum includes nonsense, frameshift, splice mutations, missense and/or small in-frame deletions, and deletions of the entire $N F-1$ gene [2]. The spontaneous mutation rate of the NF-1 gene is one of the highest known in the human genome, and is approximately 100 -fold greater than the average mutation rate [8]. About $5 \%$ of patients with Neurofibromatosis type 1 have a deletion of the entire, or nearly entire, NF-1 gene, and these patients display a more severe phenotype [9].

Mutation detection in the NF-1 gene is a complex process due to the size of the gene, the existence of pseudogenes, the lack of clustering of the mutations in a particular region of the gene, and the great variety of possible lesions, such as chromosomal abnormalities, large deletions, microdeletions, insertions, splicing mutations, nonsense and missense mutations. In NF-1 patients, the mutation detection rates rarely exceed $50 \%$. Approximately $50 \%$ of all NF-1 patients represent sporadic cases of the disease [10].

Neurofibromas are the most common feature of Neurofibromatosis type 1 . Benign nerve sheath tumours consisting of Schwann cells, fibroblasts, perineurial cells, and mast cells, may occur on the surface of the skin, along deeper peripheral nerves, and along nerve roots. Some neurofibromas, especially plexiform neurofibromas, may transform into malignant peripheral nerve sheath tumours, the lifetime risk for which is estimated at around $10 \%$. Cafe-au-lait macules, skin-fold freckling, iris Lisch nodules and melanocytic hamartomas of the iris are nontumor manifestations of NF-1. Patients may have skeletal dysplasias, scoliosis, vascular disease and epilepsy; they may also have learning disabilities, language, reading, visuospatial, neuromotor and concentration problems [11]. The 59-yearold patient in the current case presented symptoms of NF-1.

Diagnosis of Neurofibromatosis type 1 (NF-1) is based on National Institutes of Health (NIH) diagnostic criteria which are as follows: a first degree relative with NF1, six or more café-au-lait patches ( $>1.5 \mathrm{~cm}$ in adults), axillary or groin freckling, two or more neurofibromas or one plexiform neurofibroma, two or more Lisch nodules (iris hamartomas), optic pathway glioma, and distinctive bony lesions (i.e. dysplasia of the sphenoid wing, bowing of the long bone $+/$ - pseudoarthrosis) [12]. Two or more of these criteria are obligatory for a definite diagnosis. After being established, they are validated by several groups, and their utility has been proven in every day clinical practice [13]. Although they are highly specific and sensitive in the adult population, in some children, especially those with no family history of NF-1, the diagnosis can only be presumptive and delayed as many features of NF-1 develop with age [13].

Among a variety of cutaneous manifestations reported in subjects with Neurofibromatosis type 1, the most specific, e.g. café-au-lait macules, axillary/inguinal freckling (Crowe sign) and neurofibomas (dermal or plexiform), are incorporated into the set of diagnostic criteria. Less characteristic and less commonly associated with NF-1 are: increased base pigmentation, juvenile xantogranuloma, glomus tumour, melanoma, blue-red macules, pseudoatrophic macules and nevus anaemicus $[14,15]$.

As in other autosomal dominant genodermatoses, visible cutaneous signs of the NF-1 (e.g. café-au-lait macules, neurofibromas) are believed to be a result of the bi-allelic inactivation of the NF-1 gene through a 'second hit' causing loss of heterozygosity (LOH) [17].

On examination, the patient in the presented case exhibited café-au-lait spots located on the lower and upper extremities, as well as on the trunk, of longest diameter ranging from 1.5-15 cm (Fig. A, B, C, D). The classic café-au-lait spots/ macules (CALS/CALM) are well demarcated macules with smooth borders, of round to oval shape, and homogeneous tan to dark brown colour. They vary in size from $2-20 \mathrm{~cm}$. Café-au-lait macules are randomly distributed, although in opposition to ephilides, unexposed areas of the body are sites of predilection $[14,15]$.

To meet the criteria, patient needs to present with six or more café-au-lait macules $>5 \mathrm{~mm}$ in greatest diameter in 


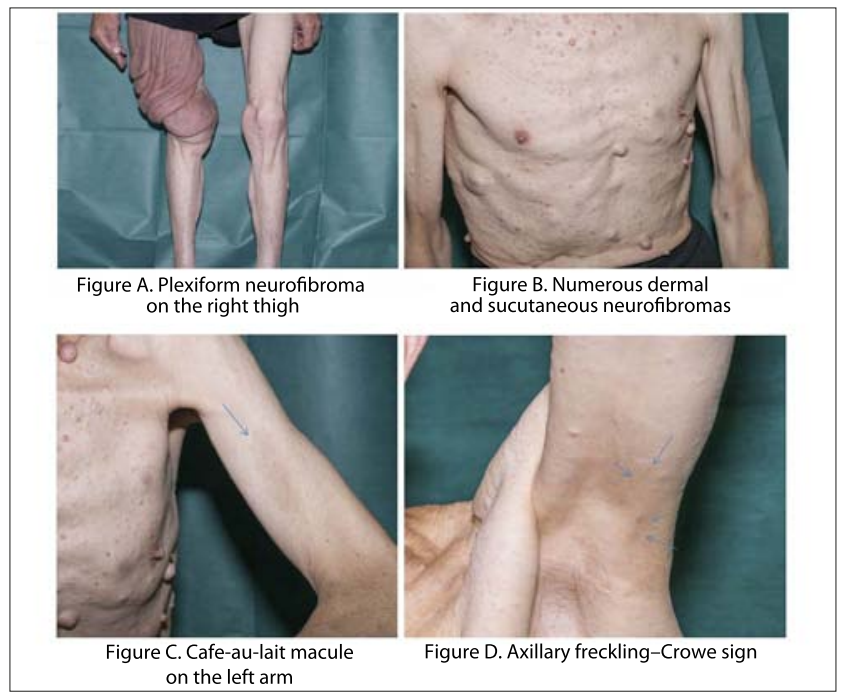

prepubertal and $>15 \mathrm{~mm}$ in greatest diameter in postpubertal individuals. Estimated incidence of one or two café-au-lait macules in the general population varies between $3-36 \%$; however, the presence of multiple (five or more) with no other sign of NF-1 does not exceed $1 \%[14,15]$. They are the earliest sign of NF-1 occurring in $99 \%$ of patients by the first year of life. If not already present, CALM development after the fourth year of life is uncommon $[14,15,16]$. In older people, CALM may fade and become obscured by neurofibromas, as in the presented case, in which four café-au-lait macules of diameter ranging from $2-15 \mathrm{~cm}$ were present.

It is noteworthy that not all individuals, even with multiple café-au-lait macules, have NF-1. In such cases, especially in the absence of other features of NF-1, differential clinicians should also exclude the following diagnosese: Mc-CuneAlbright syndrome, NF1-like syndrome (Legius syndrome), Familial café-au-lait spots, LEOPARD syndrome and Segmental NF-1 $[14,15,16,17,18,19]$.

The presented patient also had noticeable axillary fold freckling (Fig. A, B, C, D). This finding is considered to be most specific of cardinal clinical features included into NIH criteria. The so-called Crowe sign describes numerous 1-3 mm (freckle-like) macules with predilection for the axillary and inguinal regions. Usually, the freckles start to emerge by 4-6 years of age so that by the age of 7 years Crowe sign is present in almost $90 \%$ of affected individuals. Their size (1-3 mm) and localization (sun-protected areas) allows them to be distinguished from café-au-lait macules and solar-induced freckling, respectively. As mentioned above, this sign is so specific for NF-1 that only people affected by NF1-like syndrome (Legius syndrome) may present with a similar finding $[14,17,18,19,20,21,22]$.

Neurofibromas are another hallmark manifestation of NF-1 included into NIH criteria. They are benign tumours originating from the endoneurium of the peripheral nerve sheaths. Neurofibromas can occur anywhere on the body, and as in the presented case, in its full-blown expression NF-1 could present with hundreds of tumours $[14,17,18$, $19,20,21,22]$.

There are two major types of neurofibromas - cutaneous originating from the dermis and subcutaneous forms evolving from the deeper structures of skin and subcutis [14]. The tumour mass composed of Schwann cells, fibroblasts, mast cells, and perineural cells, forms soft, flesh-coloured to slightly pigmented, dome shaped or sometimes pedunculated nodules and papulo-nodules, with diameter rarely exceeding $3 \mathrm{~cm}$. These lesions do not undergo malignant transformation. In most instances, neurofibromas are asymptomatic; however, irritation and itching sensation may occur [14]. As for subcutaneous neurofibromas, they are usually firmer and less well circumscribed, and in opposition to superficial variant, they may cause tenderness, pain and bear relatively small malignant potential [14].

Neurofibromas start to evolve in the late teens or around prepubertal age, and in majority of patients are fully developed by the age of 20 ( $48 \%$ by 10 years; $84 \%$ by 20 ), although their size and number not only differ greatly among affected individuals but also the extent of the lesions shows intrafamilial variability $[17,18,19,20,21,22]$.

Finally, in the presented case, on the fronto-lateral aspect of the patient's right thigh there was visible large plexiform neurofibroma (PN), hence, along with previously described skin findings, the patient met the criteria necessary to establish diagnosis of NF-1.

In opposition to cutaneous neurofibromas, plexiform neurofibromas are usually congenital; however, only approximately $30 \%$ of cases are clinically accessible. Thus, most of the cases remain undetected unless they become apparent on imaging. Plexiform neurofibromas involve multiple nerve fascies and may extend deeply into surrounding tissues causing disfigurement. They develop from birth to the age of 18 years. Superficial forms might be surmounted by hyperpigmented skin or overlaid by hypertrichosis and feel like a 'bag of worms' upon palpation. Plexiform neurofibromas also have the potential for malignant transformation into MPNST (Malignant peripheral nerve sheath), which occurs in about $10 \%$ of patients $[14,17,18,19,20,21,22]$.

In the presented case, the large plexiform neurofibroma was not operated on because of lung cancer.

\section{CONCLUSIONS}

The pathogenic mutation p.Arg $1769^{*}$ in one of the alleles of NF1 gene was the cause of the neurofibromatosis in the presented patient. Neurofibromatosis type 1 is an inherited genetic condition which predisposes to high risk of tumour formation. In this case, the patient exhibited many of the characteristic symptoms of NF-1, most of them in a severe form.

\section{REFERENCES}

1. Savar A, Cestari DM. Neurofibromatosis Type I: Genetics and Clinical Manifestations. Seminars in Ophthalmology. 2008; 23: 45-51.

2. Firth H V, Hurst J A, Hall J G. Oxford desk reference. Clinical genetics. Oxford university press. New York, 2010.

3.http://atlasgeneticsoncology.org/Genes/NF1ID134.html

4. Marchuk D, Saulino A, Tavakkol R, Swaroop M, Wallace M, Anderson L, Mitchell A, Gutmann D, Boguski M, Collins F. cDNA cloning of the type 1 neurofibromatosis gene: complete sequence of the NF1 gene product. Genomics. 1991; 11:931- 940.

5.http://www.genecards.org/cgi-bin/carddisp.pl?gene=NF1 (access: 2014.11.20).

6. Yohay $\mathrm{K} \mathrm{H}$. The genetic and molecular pathogenesis of NF1 and NF2. Semin Pediatr Neurol. 2006; 13: 21-26.

7. Dasgupta B, Dugan LL, Gutmann DH. The neurofibromatosis 1 gene product neurofibromin regulates pituitary adenylate cyclase- activating polypeptide-mediated signaling in astrocytes. J Neurosci. 2003; 23: 8949-8954. 
8. Shen MH, Harper PS, Upadhyaya M. Molecular genetics of neurofibromatosis type 1 (NF1). J Med Genet. 1996; 33: 2-17.

9. Williams VC, Lucas J, Babcock MA, Gutmann DH, Korf B, Maria BL. Neurofibromatosis Type 1 Revisited. Pediatrics. 2009; 123: 124-133.

10. Messiaen LM, Callens T, Mortier G, Beysen D, Vandenbroucke I, Van Roy N, Speleman F, De Paepe A. Exhaustive mutation analysis of the NF1 gene allows identification of $95 \%$ of mutations and reveals a high frequency of unusual splicing defects. Hum Mutat. 2000; 15: 541-555.

11. Korf BR. The phakomatoses. Clinics in Dermatology. 2005; 23: 78-84.

12. National Institutes of Health Consensus Development Conference Statement: Neurofibromatosis. Arch Neurol Chicago. 1988; 45: 575-578.

13. DeBella K, Szudek J, Friedman JM. Use of the national institutes of health criteria for diagnosis of neurofibromatosis 1 in children. Pediatrics. 2000; 105(3 Pt1): 608-614.

14. Boyd KP, Korf BR, Theos A. Neurofibromatosis type 1. J Am Acad Dermatol. 2009; 61(1): 1-14.

15. Cohen JB, Janniger CK, Schwartz RA. Café-au-lait spots. Cutis. 2000; 66(1): $22-24$
16. Jouhilahti EM, Peltonen S, Heape AM, Peltonen J. The pathoetiology of neurofibromatosis 1. Am J Pathol. 2011; 178(5): 1932-1939.

17. DeBella K, Szudek J, Friedman JM. Use of the national institutes of health criteria for diagnosis of neurofibromatosis 1 in children. Pediatrics. 2000; 105(3 Pt1): 608-614.

18. Ferner RE, Huson SM, Thomas N, et al. Guidelines for the diagnosis and management of individuals with neurofibromatosis 1 (NF1). J Med Genet. 2007; 44: 81-88.

19. Williams VC, Lucas J, Babcock MA, Gutmann DH, Korf B, Maria BL. Neurofibromatosis type 1 revisited. Pediatrics. 2009; 123: 124-133.

20. Ferner RE. The neurofibromatoses. Pract Neurol. 2010; 10(2): 82-93.

21. Tsao H, Luo S. Neurofibromatosis and Tuberous Sclerosis. In: Bolognia JL, Jorizzo JL, Schaffer JV. Dermatology, 3rd Edition, Philadelphia, Elsevier Saunders, 2012.p.925-933.

22. Ferner RE. Neurofibromatosis 1 and neurofibromatosis 2 : a twenty first century perspective. Lancet Neurol. 2007; 6(4): 340-351. 